\title{
VARIATIONS ON THE ITÔ-MICHLER THEOREM ON CHARACTER DEGREES
}

\author{
GABRIEL NAVARRO
}

1. Introduction. Some of the most important problems in the representation theory of finite groups deal with character degrees and primes. For instance, the McKay, Alperin weight, Dade's or Brauer's height zero conjectures all have as key ingredients a prime $p$ and the degrees of the irreducible complex characters of a finite group $G$. (As usual, we denote by $\operatorname{Irr}(G)$ this set of the irreducible characters of $G$, that is, the set of traces of its irreducible complex representations. The degree of $\chi$ is $\chi(1)$.)

But, before some of these problems were even proposed, there was a simple fundamental question that had be solved: When is it true for all $\chi \in \operatorname{Irr}(G)$ that $p$ does not divide $\chi(1)$ ?

If a finite group has a normal abelian Sylow $p$-subgroup, then it can be proved that $p$ does not divide the degrees of its irreducible characters using standard character theory. The converse was addressed in the 1950's by Itô and solved for solvable groups [13]. In fact, it is not difficult to show that this converse holds for every finite group if it holds for the finite simple groups.

Thirty years later, Michler was able to completely solve the problem using the recently announced classification of finite simple groups [21]. His task was to show that a non-abelian finite simple group $G$ of order divisible by $p$ possesses an irreducible character $\chi \in \operatorname{Irr}(G)$ of degree divisible by $p$. In fact, for $p>3$, Michler proved more than that: $G$ has a $p$-defect zero character $\chi$, that is, an irreducible character such that $\chi(1)_{p}=|G|_{p}\left(n_{p}\right.$ is the largest power of $p$ dividing $\left.n\right)$. We believe

2010 AMS Mathematics subject classification. Primary $20 \mathrm{C} 15$.

Keywords and phrases. Characters, finite groups, Ito-Michler theorem.

Research partially supported by the Ministerio de Educación y Ciencia, grant No. MTM2013-40464-P.

Received by the editors on February 14, 2014, and in revised form on October 13, 2014.

DOI:10.1216/RMJ-2016-46-4-1363

Copyright (C)2016 Rocky Mountain Mathematics Consortium 
that this is one of the first applications of the classification to character theory.

Next is the central theorem on which we shall be building here.

Theorem $1.1([\mathbf{1 3}, \mathbf{2 1}])$. Let $G$ be a finite group, let $p$ be a prime and let $P \in \operatorname{Syl}_{p}(G)$. Then every $\chi \in \operatorname{Irr}(G)$ has degree not divisible by $p$ if and only if $P \triangleleft G$ and $P$ is abelian.

This survey deals with several variations of this theorem involving blocks, fields, group actions, Brauer characters or permutation characters, which are put together for the first time. We review these variations with the hope that some of the ideas here, applied to other theorems or conjectures, might perhaps suggest new results or reveal new insights.

Before we proceed, let us mention a dual result, which is also important in this survey. When talking about Itô and Michler's theorem, it is natural to mention Thompson's theorem on character degrees [37]. This is an example of a sort of wonderful duality that we sometimes have in mathematics.

Theorem 1.2 ([37]). Let $G$ be a finite group, let $p$ be a prime and let $P \in \operatorname{Syl}_{p}(G)$. Then every nonlinear $\chi \in \operatorname{Irr}(G)$ has degree divisible by $p$ if and only if $G$ has a normal p-complement $K$ and $\mathbf{C}_{K^{\prime}}(P)=1$.

Thompson's theorem is not usually stated as above. Thompson proved that, if every nonlinear $\chi \in \operatorname{Irr}(G)$ has degree divisible by $p$, then $G$ has a normal $p$-complement while the group-theoretical characterization is due to Gow and Humphreys [7]. It should be remarked that the difficulty of Thompson's theorem cannot be compared to that of Itô and Michler's in the sense that, in the latter, the classification of finite simple groups must be invoked.

Throughout, $p$ is a fixed prime. The notation for ordinary character theory follows [9] and the notation for modular theory [23].

2. Blocks. We begin with blocks. There are many ways to introduce Brauer $p$-blocks. Since the main actor of these notes is characters, we stick to characters as much as possible. Write $G_{p^{\prime}}$ for the set of elements 
of $G$ whose order is not divisible by $p$. We link $\alpha, \beta \in \operatorname{Irr}(G)$ if

$$
\sum_{x \in G_{p^{\prime}}} \alpha(x) \beta\left(x^{-1}\right) \neq 0,
$$

and the connected components of this linking are the $p$-blocks of $G$. The principal p-block $B_{0}(G)$, the most important block, is the connected component of the trivial character. In fact, the principal block consists of the set of characters $\chi \in \operatorname{Irr}(G)$ such that

$$
\sum_{x \in G_{p^{\prime}}} \chi(x) \neq 0,
$$

since the trivial character is linked to every character in the principal block [23, Corollary (3.25)]. The following is still open.

Conjecture 2.1. Let $P \in \operatorname{Syl}_{p}(G)$. Then every $\chi \in B_{0}(G)$ has degree not divisible by $p$ if and only if $P$ is abelian.

Of course, this is Brauer's height zero conjecture for the principal blocks, and we shall say something else about this later on. For the time being, let us comment that Tiep and this author have proved the case $p=2$ of Conjecture 2.1 ([29]).

Character theorists love to discover group theoretical properties in the character table, and Conjecture 2.1 gives a beautiful method of checking whether a group has abelian Sylow subgroups from the character table. (A related way to check this fact has recently been given in [26].)

We remark that if, in Itô and Michler's theorem, we restrict our attention to the characters in the principal block, then the commutativity of $P$ is characterized while the normality of $P$ is lost. An ongoing problem has been finding the exact subset of $\operatorname{Irr}(G)$ that characterizes the normality of $P$ (and forgets about its commutativity). The solution is offered in the last variation in these notes.

What does the height zero conjecture assert in general? Recall that $G$ has a $p$-subgroup $D$ associated to every $p$-block $B$ (uniquely determined up to $G$-conjugacy), which has deep connections with the characters in the block. These $p$-subgroups are the defect groups of the block. For instance, a block $B$ consists of exactly one character $\{\chi\}$ if 
and only if $D=1$. Another connection with characters is that $\chi(x)=0$ if $x_{p}$ does not lie in a $G$-conjugate of $D$ and $\chi \in B$. Also, the minimum of $\left\{\chi(1)_{p} \mid \chi \in B\right\}$ is $|G|_{p} /|D|$.

Conjecture 2.2 (Brauer's height zero). Let $B$ be a p-block with defect group $D$. Then the degrees of all the irreducible characters in $B$ have the same p-part if and only if $D$ is abelian.

The if part now is known by the results from Kessar and Malle [15]. The only if part, which remains open, is even deeper

We take this opportunity to mention another recent conjecture on character degrees which appears in a joint paper of this author and Malle [17]. As Thompson's theorem is the dual of Itô and Michler's theorem, we consider the following as the dual of Brauer's height zero conjecture. Nilpotent blocks were introduced by Broué and Puig in [1] and are considered the most natural blocks from the local point of view. For instance, the principal block is nilpotent if and only if $G$ has a normal $p$-complement.

Conjecture 2.3. Let $B$ be a block. Then the degrees of all of the irreducible characters in $B$ have the same $p^{\prime}$-part if and only if $B$ is nilpotent.

Hence, nilpotent blocks and blocks with an abelian defect group are like opposite faces of the same coin (from this point of view).

The following theorem, due to Isaacs and Smith [12, Corollary 3], is the dual of Conjecture 2.1, and it is a particular case of Conjecture 2.3.

Theorem 2.4. $G$ has a normal p-complement if and only if every nonlinear irreducible character in $B_{0}(G)$ has degree divisible by $p$.

Theorem 2.4 can be generalized further. The following is a result of Pahlings [35]. Recall that, if $\chi \in \operatorname{Irr}(G)$ and $\phi \in \operatorname{IBr}(G)$, then $d_{\chi \phi}$ is the decomposition number and

$$
\Phi_{\phi}=\sum_{\chi \in \operatorname{Irr}(G)} d_{\chi \phi} \chi
$$


is the projective indecomposable character. We write $\operatorname{Irr}\left(\Phi_{1}\right)$ for the irreducible constituents of $\Phi_{1}$, and we notice that $\operatorname{Irr}\left(\Phi_{1}\right) \subseteq B_{0}(G)$.

Theorem 2.5. Let $G$ be a finite group. Then every nonlinear $\chi$ in $\operatorname{Irr}\left(\Phi_{1}\right)$ has degree divisible by $p$ if and only if $G$ has a normal $p$ complement.

Theorem 2.5 suggests a refinement of Conjecture 2.1, which is wrong: if every irreducible constituent of $\operatorname{Irr}\left(\Phi_{1}\right)$ has degree not divisible by $p$, it is false that $G$ has abelian Sylow $p$-subgroups. The symmetric group $S_{4}$ with $p=2$ is already a counterexample.

3. Brauer characters. A typical way to generalize a theorem on ordinary characters is to ask to what extent the same result holds for $p$-Brauer characters. So the variation of Itô and Michler's theorem with respect to Brauer characters would be: for some prime $q$, when is it true that, for every $\phi \in \operatorname{IBr}(G)$, the degree $\phi(1)$ is not divisible by $q$ ? Although this question has been studied, it seems to us that the right theorem (if such a theorem exists) has not yet been found. In our opinion, it is very difficult to find general good theorems on the degrees of Brauer characters, except perhaps in two cases: if our group $G$ is $p$-solvable or if our interest is in $q=p$.

The following is another consequence of the classification [21].

Theorem 3.1. Suppose that $G$ is a finite group, and let $P \in \operatorname{Syl}_{p}(G)$. Then every $\phi \in \operatorname{IBr}(G)$ has degree not divisible by $p$ if and only if $P \triangleleft G$.

In Section 2, we searched for a criterion for $P$ to be normal in $G$, and Theorem 3.1 provides one by using Brauer character degrees. Brauer character degrees, except in $p$-solvable groups, are not known to be detectable in the ordinary character table (this is by itself an important problem). Let us mention that there is a clever elementary proof by Okuyama for $p=2$ of the previous theorem [23, Theorem (2.33)].

For solvable groups, the question of when all the irreducible Brauer characters have $q^{\prime}$-degree was studied by Manz and Wolf (see [20, Theorem (13.8)]), and some of the structure of these groups was revealed. 
Problem 3.2. Let $G$ be a finite $p$-solvable group, and let $q$ be a prime. Find a group theoretical characterization of when every irreducible $p$ Brauer character of $G$ has degree not divisible by $q$. Is it true in this case that every $p$-regular conjugacy class of $G$ meets $\mathbf{N}_{G}(Q)$, where $Q \in \operatorname{Syl}_{q}(G)$ ?

Proof. Problem 3.2 has recently been proved by Lewis and Tong-Viet [16].

We find it interesting that the question in Problem 3.2 has an affirmative answer in the case where $p=q$ for every finite group $G$. This follows from Theorem 3.1 and a deep result in [4]: the only subgroup $H$ of $G$ whose $G$-conjugates contain all the prime power order elements is $G$ itself.

Thompson's theorem for Brauer characters was proved in [7], while the group-theoretical characterization was given in [34]. All of these results need some solvability assumptions.

Theorem 3.3. Suppose that $G$ is $p$-solvable, and suppose that $p \neq q$. Then, every nonlinear irreducible $p$-Brauer character of $G$ has degree divisible by $q$ if and only if $G$ has a normal $q$-complement $K$ and $\mathbf{C}_{K^{\prime}}(Q)$ is a p-group, where $Q \in \operatorname{Syl}_{q}(G)$.

Another type of duality with respect to Itô and Michler's theorem is given in a recent theorem in [28].

Theorem 3.4. Suppose that $p>2$. Then every $1 \neq \phi \in \operatorname{IBr}(G)$ has degree divisible by $p$ if and only if $\mathbf{N}_{G}(P)=P$.

Hence, we see that the fact that a group has a self-normalizing Sylow $p$-subgroup, at least for $p$ odd, can be detected from the degrees of the irreducible Brauer characters. (We do not know of any other similar result for $p=2$.) But, in fact, if $p$ is odd, we can check whether $\mathbf{N}_{G}(P)=P$ from the ordinary character table [32]. For $p=2$, a method is proposed in [22]. 
4. Fields. The study of fields of values of characters is a classical part of character theory. Using them, we can obtain generalizations of Itô and Michler's or of the Thompson theorem.

If $\mathbb{Q} \subseteq F \subseteq \mathbb{C}$ is a subfield, then we write

$$
\operatorname{Irr}_{F}(G)=\{\chi \in \operatorname{Irr}(G) \mid \chi(g) \in F \quad \text { for all } g \in G\} \text {. }
$$

A few important cases follow from equation (4.1):

$$
\operatorname{Irr}_{\mathbb{Q}}(G), \quad \operatorname{Irr}_{\mathbb{R}}(G), \quad \operatorname{Irr}_{\mathbb{Q}_{p^{\prime}}}(G),
$$

that is, the subset of rational, real or $p$-rational characters of $G$. (A character is $p$-rational if its values lie in some cyclotomic field $\mathbb{Q}_{n}$ for some $n$ not divisible by $p$.)

When dealing with real characters, $p=2$ is the natural prime. The following result was proven [3]. Recall that a 2-group is of ChillagMann type [2] if the only irreducible real characters of $P$ are linear.

Theorem 4.1. Let $G$ be a finite group, and let $P \in \operatorname{Syl}_{2}(G)$. Then every $\chi \in \operatorname{Irr}_{\mathbb{R}}(G)$ has odd degree if and only if $P \triangleleft G$ and $P$ is of Chillag-Mann type.

Marinelli and Tiep have proved that it is possible to replace $\operatorname{Irr}_{\mathbb{R}}(G)$ in the previous theorem for even a smaller subset: the real characters with Frobenius-Schur indicator equal to 1 [19].

For some time, we have wondered if there was an analogous result of Theorem 4.1 for $p$ odd. Since groups of odd order do not have nontrivial irreducible real characters, it is natural that $\mathbf{O}^{2^{\prime}}(G)$, the smallest normal subgroup of $G$ with odd index, has a role in the solution.

The following is a combination of the main results of $[\mathbf{1 1}, \mathbf{3 8}]$ (which also extends the difficult part of Theorem 4.1).

Theorem 4.2. Let $p$ be a prime. Assume that every $\chi \in \operatorname{Irr}_{\mathbb{R}}(G)$ has degree not divisible by $p$. Then $\mathbf{O}^{2^{\prime}}(G)$ has a normal Sylow $p$-subgroup.

Next we present one more Itô-Michler type theorem, by considering $p$-rational characters only [30]. 
Theorem 4.3. Assume that every $\chi \in \operatorname{Irr}_{\mathbb{Q}_{p^{\prime}}}(G)$ has degree not divisible by $p$. Then $G$ has a normal Sylow p-subgroup.

We now return to Thompson's theorem. It is pleasant that, when introducing fields of values, we are able to obtain natural characterizations. The following appears in $[\mathbf{2 5}, \mathbf{2 7}]$.

Theorem 4.4. Let $G$ be a finite group, and let $P \in \operatorname{Syl}_{2}(G)$. Then the following conditions are equivalent:

(a) Every nonlinear $\chi \in \operatorname{Irr}_{\mathbb{R}}(G)$ has even degree.

(b) Every nonlinear $\chi \in \operatorname{Irr}_{\mathbb{Q}}(G)$ has even degree.

(c) $G$ has a normal 2-complement.

Although the equivalence of (a) and (c) is elementary, the equivalence between (a) and (b) is much deeper and requires the classification of finite simple groups. In addition, a $p$ odd version of part of the above result can be obtained if we replace $\mathbb{Q}$ by $\mathbb{Q}_{p}$, the cyclotomic field [27].

Without going into detail, all these theorems need general results of the following type.

Problem 4.5. Suppose that $N \triangleleft G$ and $\theta \in \operatorname{Irr}_{F}(N)$, where $\mathbb{Q} \subseteq F \subseteq \mathbb{C}$ is a field. Find conditions on $N, \theta$ or $G / N$ to guarantee that there exists $\chi \in \operatorname{Irr}(G \mid \theta)$ which is $F$-valued.

5. Minimal situations. Minimal situations constitute a central subject in group theory. If we set

$$
\operatorname{cd}_{p^{\prime}}(G)=\left\{\chi(1) \mid \chi \in \operatorname{Irr}_{p^{\prime}}(G)\right\},
$$

where $\operatorname{Irr}_{p^{\prime}}(G)$ is the set of irreducible characters of $G$ of degree not divisible by $p$, then Itô-Michler considers the case $\operatorname{cd}(G)=\operatorname{cd}_{p^{\prime}}(G)$. In search for general patterns, in [10], we consider the "next" case:

$$
\operatorname{cd}(G)=\operatorname{cd}_{p^{\prime}}(G) \cup\{a\} .
$$

Among other facts, the following was proven. 
Theorem 5.1. Let $p$ be a prime, let $G$ be a finite group, and let $P \in \operatorname{Syl}_{p}(G)$. If $\operatorname{cd}(G)=\operatorname{cd}_{p^{\prime}}(G) \cup\{a\}$, then $P$ is metabelian.

Although we have not checked as many examples as possible, this suggests to us that, perhaps, if $G$ is a finite group with Sylow $p$ subgroup $P$, then $\mathrm{dl}(P)-1$ is bounded by the number of irreducible character degrees which are divisible by $p$. Some analogous results might even be true for blocks, heights and defect groups.

The Itô-Michler theorem offers, as we now know, two adjectives on $P$ : normal and abelian. In the situation where $\operatorname{cd}(G)=\operatorname{cd}_{p^{\prime}}(G) \cup\{a\}$, we have that $P$ is metabelian, by Theorem 5.1 , but it seems that nothing can be said about "how normal" $P$ is in $G$. For instance, if we consider dihedral groups, we have that

$$
\operatorname{cd}\left(D_{2(2 n+1)}\right)=\{1,2\},
$$

while the Sylow 2-subgroup of this group is self-normalizing of size 2.

The following minimal situation was studied and classified [6].

Theorem 5.2. Let $p$ be a prime, let $G$ be a finite group, and let $P \in \operatorname{Syl}_{p}(G)$. If $\operatorname{Irr}(G)=\operatorname{Irr}_{p^{\prime}}(G) \cup\{\chi\}$, then $P$ is normal in $G$ or $\mathbf{N}_{G}(P)$ is maximal.

This theorem suggests that the number of characters of degree divisible by $p$ is perhaps bounded by the length of any saturated chain of subgroups from $\mathbf{N}_{G}(P)$ to $G$.

What are the minimal situations in Thompson's theorem? If the Itô-Michler hypothesis is

$$
\operatorname{cd}(G)=\operatorname{cd}_{p^{\prime}}(G),
$$

then Thompson's is $\operatorname{cd}_{p^{\prime}}(G)=\{1\}$. Hence the "next" situation would be

$$
\operatorname{cd}_{p^{\prime}}(G)=\{1, a\}
$$

or even the stronger hypothesis, dual of Theorem 5.2:

$$
\operatorname{Irr}(G)=\operatorname{Irr}\left(G / G^{\prime}\right) \cup\{\chi\} .
$$

The groups satisfying this latter condition have been classified in [14]. 
Problem 5.3. What can be said about the groups $G$ such that $\left|\mathrm{cd}_{p^{\prime}}(G)\right|=2$ ?

It seems that, for $p>3$, groups with $\left|\operatorname{cd}_{p^{\prime}}(G)\right|=2$ are $p$-solvable. (There seem to be many non-solvable groups satisfying this condition for $p=2$.)

6. Actions and $q^{\prime}$-degree characters. When a finite group $A$ acts coprimely on another finite group $G$, the set of $A$-invariant objects of $G$ has a rich structure. Many theorems on groups or characters admit an $A$-version. As an example, the following is the $A$-version of Sylow's theorems. Every $A$-invariant $p$-subgroup of $G$ is contained in an $A$ invariant Sylow $p$-subgroup of $G$, and any two are $\mathbf{C}_{G}(A)$-conjugate. The following is the version of the Itô-Michler theorem from the point of view of a coprime action. Unfortunately, it only holds under a certain solvability hypothesis [22].

Theorem 6.1. Suppose that $A$ acts coprimely on a p-solvable group $G$, let $P \in \operatorname{Syl}_{p}(G)$ be A-invariant, and let $C=\mathbf{C}_{G}(A)$. Then every $\chi \in \operatorname{Irr}_{A}(G)$ has a degree not divisible by $p$ if and only if $C$ normalizes $P$ and $\mathbf{C}_{P^{\prime}}(A)=1$.

In a coprime action, the $A$-invariant characters extend to the semidirect product $G A$ characters that have degree coprime with $|A|$. As a further generalization, this suggests looking at the set of irreducible characters of a finite group whose degrees are not divisible by some prime $q$ as the basic object. Again, this needs a solvability hypothesis and was proven $[\mathbf{3 3}]$.

Theorem 6.2. Suppose that $G$ is a solvable group. Let $P \in \operatorname{Syl}_{p}(G)$ and $Q \in \operatorname{Syl}_{q}(G)$ be such that $P Q=Q P$. Then, every $\chi \in \operatorname{Irr}_{q^{\prime}}(G)$ has degree not divisible by $p$ if and only if $\mathbf{N}_{G}(Q) \subseteq \mathbf{N}_{G}(P)$ and $\mathbf{C}_{P^{\prime}}(Q)=1$.

It would be apropos to comment on Thompson's $A$-version here, but it does not seem that the right theorem (if it exists) has been found yet. 
Problem 6.3. Suppose that $A$ acts coprimely on $G$. Is it possible to give a group-theoretical characterization of when every nonlinear character $\chi \in \operatorname{Irr}_{A}(G)$ has degree divisible by $p$ ? If $G$ is any finite group and $p$ and $q$ are primes, is it possible to give a group-theoretical characterization of when every nonlinear character in $\operatorname{Irr}_{q^{\prime}}(G)$ has degree divisible by $p$ ?

Part of the difficulty of solving Problem 6.3 is that what seems to be the most natural generalization of Thompson's theorem does not hold: if $A$ acts coprimely on $G$ and $p$ divides $\chi(1)$ for all nonlinear $\chi \in \operatorname{Irr}_{A}(G)$, then it is false that $C=\mathbf{C}_{G}(A)$ has a normal $p$ complement. If $K$ is the extra-special group of order $7^{3}$ and exponent 7 , then Aut $(K)$ has several conjugacy classes of cyclic subgroups $U$ of order 6. It is possible to choose $U$ such that, in the semidirect product $K U$, if we take $G=K H$ and consider the action of $A$ on $G$ (where $U=H \times A, H$ has order 3 and $A$ has order 2), then every nonlinear irreducible $A$-invariant of $K$ has degree divisible by $p=7$ while $C=\mathbf{C}_{G}(A)$ is isomorphic to the Frobenius group of order 21 .

7. Characters over a character of a normal subgroup. Suppose that $N \triangleleft G$, and let $\theta \in \operatorname{Irr}(N)$ be $G$-invariant. Now our key object is $\operatorname{Irr}(G \mid \theta)$, that is, the set of the irreducible constituents of the induced character $\theta^{G}$ (or equivalently, the set of the irreducible characters $\chi$ of $G$ such that $\left.\left[\chi_{N}, \theta\right] \neq 0\right)$. As is well known, the numbers $\chi(1) / \theta(1)$ are the degrees of the irreducible representations of a twisted group algebra $\mathbb{C}_{\alpha}[G / N]$ naturally associated with $\theta$. (These numbers are also called "projective degrees.")

It would be a mistake to underestimate the importance of these "projective degrees" in character theory. This is especially true currently since it is now believed that all the conjectures mentioned at the beginning of these notes should have a "twisted version." So, returning to the Itô-Michler theorem: What happens if every $\chi \in \operatorname{Irr}(G \mid \theta)$ has a degree not divisible by $p$ ? What we would like to prove is that the Sylow $p$-subgroups of $G / N$ are abelian. It is not that well known, but this fact is implied by Brauer's height zero conjecture.

The if direction of the height zero conjecture was reduced to quasisimple groups by Berger and Knörr in the 1980's, and, as we have already said, it has now been completed by Kessar and Malle [15]. The 
only if direction is even harder. It was proven by Gluck and Wolf [5] for $p$-solvable groups. (The if direction is almost trivial for $p$-solvable groups.) In fact, almost all the work they did was concentrated in proving the following deep theorem.

Theorem 7.1 ([5]). Let $p$ be a prime, let $G$ be a finite $p$-solvable group, let $N \triangleleft G$, and let $\theta \in \operatorname{Irr}(N)$. If every $\chi \in \operatorname{Irr}(G \mid \theta)$ has degree not divisible by $p$, then $G / N$ has abelian Sylow p-subgroups.

To drop the hypothesis that $G / N$ is $p$-solvable in the previous theorem has been a tough problem for many years. In fact, this was one of the obstacles to proving Brauer's height zero conjecture. Fortunately, this theorem was recently proved [31].

Problem 7.2. Let $p$ be a prime, let $G$ be a finite group, let $N \triangleleft G$, and let $\theta \in \operatorname{Irr}(N)$ be $G$-invariant. Is there a group-theoretical characterization of when every $\chi \in \operatorname{Irr}(G \mid \theta)$ has degree not divisible by $p$ ?

The dual version of Problem 7.2 would be to characterize when, for every $\chi \in \operatorname{Irr}(G \mid \theta)$, we have that $p$ divides $\chi(1) / \theta(1)$.

8. Permutation characters. We mentioned in Section 2 that, for some time, we have been looking for the exact subset of $\operatorname{Irr}(G)$ that characterizes the normality of $P$ (and forgets its commutativity). We have recently found this subset.

If $H \subseteq G$, let $\operatorname{Irr}\left(\left(1_{H}\right)^{G}\right)$ be the set of the irreducible constituents of the permutation character $\left(1_{H}\right)^{G}$. The following is the main result of $[18]$.

Theorem 8.1. Let $G$ be a finite group, $p$ a prime and $P \in \operatorname{Syl}_{p}(G)$. Then $P \triangleleft G$, if and only every $\chi \in \operatorname{Irr}\left(\left(1_{P}\right)^{G}\right)$ has degree not divisible by $p$.

Theorem 8.1 again brings up the subject of how to find the irreducible constituents of $\left(1_{P}\right)^{G}$ in the character table. This is another 
interesting problem on its own. Koshitani pointed out to us that Theorem 8.1 was previously conjectured [36].

As our final result, we look for a theorem similar to Thompson's with respect to permutation characters.

Theorem 8.2. Suppose that $G$ has a p-complement $H$. Then every nonlinear irreducible character in $\operatorname{Irr}\left(\left(1_{H}\right)^{G}\right)$ has degree divisible by $p$ if and only if $H \triangleleft G$.

Proof. We have that $\Phi_{1}=\left(1_{H}\right)^{G}$ by [23, Theorem (2.27)]. Now, Theorem 2.5 applies.

Acknowledgments. Finally, we would like to thank M. Isaacs for many conversations on this paper, and for helping us to improve its presentation.

\section{REFERENCES}

1. M. Broué and L. Puig, A Frobenius theorem for blocks, Invent. Math. 56 (1980), 117-128.

2. D. Chillag and A. Mann, Nearly odd-order and nearly real finite groups, Comm. Alg. 26 (1998), 611-620.

3. S. Dolfi, G. Navarro and P.H. Tiep, Primes dividing the degrees of the real characters, Math. Z. 259 (2008), 755-774.

4. B. Fein, W.M. Kantor and M. Schacher, Relative Brauer groups, II, J. reine angew Math. 328 (1981), 39-57.

5. D. Gluck and T.R. Wolf, Brauer's height conjecture for p-solvable groups, Trans. Amer. Math Soc. 282 (1984), 137-152.

6. D. Goldstein, R.M. Guralnick, M.L. Lewis, A. Moretó, G. Navarro and P. H. Tiep, Groups with exactly one irreducible character of degree divisible by $p$, Alg. Num. Theor. 8 (2014), 397-428.

7. R. Gow and J.F. Humphreys, Normal p-complements and irreducible representations, J. Lond. Math. Soc. 11 (1975), 308-312.

8. M. Isaacs, Characters of $\pi$-separable groups, J. Alg. 86 (1984), 98-128.

9. Character theory of finite groups, Amer. Math Soc. Chelsea Publishing, Providence, RI, 2006.

10. M. Isaacs, A. Moretó, G. Navarro and P. H. Tiep, Groups with just one character degree divisible by a given prime, Trans. Amer. Math. Soc. 361 (2009), $6521-6547$.

11. M. Isaacs and G. Navarro, Groups whose real irreducible characters have degrees coprime to p, J. Alg. 356 (2012), 195-206. 
12. M. Isaacs and S.D. Smith, A note on groups of p-lenght 1, J. Alg. 38 (1976), $531-535$.

13. N. Itô, Some studies on group characters, Nagoya Math. J. 2, (1951), 17-28.

14. L. Kazarin and Y. Berkovich, On Thompson's theorem, J. Alg. 220 (1999), $574-590$.

15. R. Kessar and G. Malle, Quasi-isolated blocks and Brauer's height zero conjecture, Ann. Math. 178 (2013), 321-384.

16. M. Lewis and H. Tong-Viet, Brauer characters of $q^{\prime}$-degree, Proc. Amer. Math Soc., to appear.

17. G. Malle and G. Navarro, Blocks with equal height zero degrees, Trans. Amer. Math. Soc. 363 (2011), 6647-6669.

18. , Characterizing normal Sylow p-subgroups by character degrees, J. Alg. 370 (2012), 402-406.

19. S. Marinelli and P.H. Tiep, Zeros of irreducible characters of finite groups, Alg. Num. Theor. 7 (2013), 567-593.

20. O. Manz and T.R. Wolf, Representations of solvable groups, LMS Lect. Note Ser. 185, Cambridge University Press, Cambridge, 1993.

21. G.O. Michler, Brauer's conjectures and the classification of finite simple groups, in Lect. Notes Math. 1178, Springer, Berlin, 1986.

22. G. Navarro, Actions and invariant character degrees, J. Alg. 160 (1993), $172-178$.

23. Characters and blocks of finite groups, Cambridge University Press, Cambridge, 1998.

24. The McKay conjecture with Galois automorphisms, Ann. Math. 160 (2004), 1129-1140.

25. G. Navarro, L. Sanus and P.H. Tiep, Real characters and degrees, Israel J. Math. 171 (2009), 157-173.

26. G. Navarro, R. Solomon and P.H. Tiep, Abelian Sylow subgroups in a finite group, II, J. Alg. 421 (2015), 3-11.

27. G. Navarro and P.H. Tiep, Degrees of rational characters of finite groups, Adv. Math. 224 (2010), 1121-1142.

28. , On p-Brauer characters of $p$-degree and self-normalizing Sylow $p$ subgroups, J. Group Theor. 13 (2010), 785-797.

29. Brauer's height zero conjecture for the 2-blocks of maximal defect, J. reine angew. Math. 669 (2012), 225-247.

30. Degrees and p-rational characters, Bull. Lond. Math. Soc. 44 (2012), 1246-1250.

31. Characters of $p^{\prime}$-degree over normal subgroups, Ann. Math. 178 (2013), 1135-1171.

32. G. Navarro, P.H. Tiep and A. Turull, p-rational characters and selfnormalizing Sylow p-subgroups, Represent. Theor. 11 (2007), 84-94. 
33. G. Navarro and T.R. Wolf, Character degrees and local subgroups of $\pi$ separable groups, Proc. Amer. Math. Soc. 126 (1998), 2599-2605.

34. H. Pahlings, Character degrees and normal p-complements, Comm. Alg. 3 (1975), 75-80.

35. _ Normal p-complements and irreducible characters, Math. Z. 154 (1977), 243-246.

36. A.I. Saksonov, Criterion for a finite group to be p-closed in terms of the degrees of its characters, Math. Z. 42 (1987), 177-179.

37. J.G. Thompson, Normal p-complements and irreducible characters, J. Alg. 14 (1970), 129-134.

38. P.H. Tiep, Real ordinary characters and real Brauer characters, Trans. Amer. Math. Soc. 367 (2015), 1273-1312.

Departament d'Àlgebra, Facultat de Matemàtiques, Universitat de ValènCIA, 46100 Burjassot, VAlÈncia, Spain

Email address: gabriel.navarro@uv.es 